\title{
A systematic review and meta-analysis on the global prevalence of microsporidia infection among dogs: a zoonotic concern
}

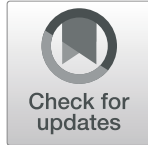

\author{
Ali Taghipour ${ }^{*}$, Saeed Bahadory ${ }^{\dagger}$ and Sasan Khazaei ${ }^{\dagger}$
}

\begin{abstract}
Background: Microsporidiosis is an emerging zoonotic disease that is considered a global public health concern. Dogs are suggested as one of potential reservoirs for transmitting the microsporidia infection to humans. However, there is little data on distribution of microsporidia in dogs. The current study aimed to evaluate the global prevalence and genetic diversity of microsporidia infection among the dog population.

Methods: We searched four major databases for studies reporting the prevalence of microsporidia in dogs until 30 May 2020. A random-effects model was used to estimate the overall and the subgroup-pooled prevalence of microsporidia across studies.

Result: Finally, a total of 32 studies (including 37 datasets) from 17 countries were included in this meta-analysis. The overall prevalence $(95 \% \mathrm{Cl})$ of microsporidia infection was estimated at $23.1 \%$ (13.5-36.8\%) using microscopic methods, 20.9\% (14.6-29\%) using serological methods, and 8.4\% (6.1-11.5\%) using molecular methods. Molecular methods showed that the highest number of reports was related to Enterocytozoon bieneusi with a pooled prevalence of $6.5 \%$ (4.9-8.7\%). Considering E. bieneusi genotypes, most studies reported the PtEb IX (10 studies) and the D (eight studies) genotypes.

Conclusion: These results emphasize the role of a dog as a reservoir host for human-infecting microsporidia. In addition, monitoring programs for human-infecting microsporidia in animals with close contact to humans should be considered.
\end{abstract}

Keywords: Microsporidia infection, Dogs, Prevalence, Meta-analysis

\section{Background}

Microsporidiosis is an emerging disease caused by opportunistic unicellular organisms called microsporidia comprising more than 200 genera with approximately 1500 species. Microsporidia can infect a broad range of vertebrate and invertebrate hosts [1-3]. Epidemiological studies over the world showed that Enterocytozoon bieneusi (E. bieneusi) and Encephalitozoon species (Enc. cuniculi, Enc. intestinalis, and Enc. hellem) are the most

\footnotetext{
* Correspondence: alitaghipor71@yahoo.com

${ }^{\dagger}$ Ali Taghipour, Saeed Bahadory and Sasan Khazaei contributed equally to this work.

Department of Parasitology, Faculty of Medical Sciences, Tarbiat Modares University, P.O. Box 14115-111, Tehran, Iran
}

common species of microsporidia reported from humans and a wide range of animals $[4,5]$. Microsporidia infection in immunocompetent individuals is mostly limited to mild or self-limiting diarrhea. This infection maybe reported from intestinal, pulmonary, ocular, muscular, and renal diseases in immunocompromised patients [69]. The main transmission routes of microsporidia seem to be fecal-oral transmission of spores from infected humans and animals through contaminated food and water together with inhalation of spores [2, 10, 11]. Human-infecting microsporidia have been frequently reported from domesticated animals, wild and laboratory animals, and birds highlighting the high probability of zoonotic transmission $[12,13]$.

(c) The Author(s). 2020 Open Access This article is licensed under a Creative Commons Attribution 4.0 International License, which permits use, sharing, adaptation, distribution and reproduction in any medium or format, as long as you give appropriate credit to the original author(s) and the source, provide a link to the Creative Commons licence, and indicate if changes were made. The images or other third party material in this article are included in the article's Creative Commons licence, unless indicated otherwise in a credit line to the material. If material is not included in the article's Creative Commons licence and your intended use is not permitted by statutory regulation or exceeds the permitted use, you will need to obtain permission directly from the copyright holder. To view a copy of this licence, visit http://creativecommons.org/licenses/by/4.0/. 
From past to present, dogs play an important role in the psychological and physiological well-being of humans, especially children and elderly individuals $[14,15]$. Nevertheless, dogs pose a potential health risk to human society, so that they can transmit more than 100 infectious zoonotic diseases to humans [16-19]. In relation to the OneHealth approach, the health of a community is connected to the health of animals and the environment [20,21]. Therefore, dogs play a major role in the transmission dynamics of microsporidia spores due to their wide niche and close contact to humans [12, 22, 23].

In recent years, a growing number of studies have been conducted on the prevalence of microsporidia infection among dogs, although there is a big gap in our knowledge in many countries. In this review, we described the global prevalence and genetic diversity of microsporidia infection in dogs.

\section{Materials and methods}

\section{Systematic search strategy}

In this review, the protocols recommended by the "preferred reporting items for systematic reviews and metaanalyses statement (PRISMA)" were followed for all eligible studies [24]. To assess the prevalence of microsporidia infection in dogs from a global scale, we performed a comprehensive systematic review and meta-analysis of literature (full text or abstracts) published online. Four major databases (PubMed, Web of Science, Scopus, and Google Scholar) were searched by two expert reviewers (S.B. and S.K.) for relevant studies published until 30 May 2020. Searching process was accomplished using $\mathrm{MeSH}$ terms alone or in combination: ("Microsporidiosis" OR "Microsporidia" OR "Microsporidium" OR "Microspora" OR "Nosema" OR "Enterocytozoon bieneusi" OR "Encephalitozoon spp.") AND ("Prevalence” OR "Epidemiology") AND ("Dog" OR "Canine"). Additionally, the references of all selected articles were handsearched to finding other relevant articles or their citations by searching in Google Scholar.

\section{Eligibility criteria, study selection, and data extraction}

Eligible articles were screened initially by title and abstract. All selected articles were imported into the EndNote X8 software (Thomson Reuters, New York, USA), and duplicated articles were checked and then removed. After removing duplicates, the full text of qualified records was retrieved and two expert investigators (S.B. and S.K.) evaluated the eligibility of the articles. Afterward, an investigator (A.T.) extracted the required information, and two others (S.B. and S.K.) rechecked them; any discrepancies of opinion or disagreement were resolved by consensus and discussion with the lead investigator (A.T.). In this systematic review, a strict protocol for the inclusion of papers was defined by our research team. So, we included articles that they had all of the following inclusion criteria: (1) peer-reviewed original research articles or short reports or letters to the editors that studied the prevalence of microsporidia in dogs; (2) the papers with full-text or abstract in English without geographical restriction; (3) papers published online from the inception up to 30 May 2020 with a digital object identifier (DOI); (4) the investigations which examined microsporidia infection via at least one of the following methods, including molecular, serology, microscopy, or coproantigen, to detect the microorganism in feces and/or serum; and (5) those papers that provided the exact total sample size and positive samples. The papers were excluded if they did not meet the abovementioned criteria. Besides, we excluded articles from further considerations with the following characteristics: (1) the articles that reported microsporidia infection from humans and animals (except dogs); (2) specimens collected from the urine and tissues of dogs; (3) investigations of dogs which had been experimentally infected for other purposes, but not prevalent; (4) immunocompromised animals; and (5) all types of review papers, case reports, and case series.

The following variables were extracted for each study: first author's last name, year of publication, country, types of samples tested (fecal and/or serum), diagnostic method, type of animals (pet or stray), the number of studied animals, and the number of animals with a positive test result. In addition, if possible, the prevalence was stratified by age ( $\leq 12$ vs $>12$ months of age) and gender of animals. Concerning molecular methods, the identified species/genotypes were also extracted. Since there were several types of dogs in the studies included, we divided dogs into (1) pet (domesticated) animals: "pet, household, sheltered or domesticated"; (2) stray animals: "stray, free-roaming or feral"; (3) unknown animals: "It is not clear whether the dogs are domesticated or stray."

\section{Study quality assessment}

The Joanna Briggs Institute (JBI) checklist was used for quality assessment of the included articles [25]. This checklist contains ten questions with four options including, yes, no, unclear, and not applicable. Briefly, a study can be awarded a maximum of one star for each numbered item. The papers with a total score of 4-6 and 7-10 points were specified as the moderate and high quality, respectively. Based on the obtained score, the authors have decided to include (4-10 points) and exclude ( $\leq 3$ points) the papers.

\section{Data synthesis and statistical analysis}

Comprehensive Meta-Analysis (V2.2, Bio stat) software was used to perform analysis. To minimize the biases, 
we calculated the prevalence of microsporidia infection (the pooled prevalence and $95 \%$ confidence interval $(\mathrm{CI})$ ) for individual diagnostic methods (molecular, serology, microscopy methods) using the random-effects model (REM). The REM allows for a distribution of true effect sizes between published studies. Moreover, using subgroup analyses, the pooled prevalence of microsporidia infection was estimated according to the continents, countries, and type of animals. An odds ratio (OR) (and the corresponding 95\% $\mathrm{CI}$ ) was calculated using REM for each study to assess the association between microsporidia prevalence and risk factors such as age (animals aged 12 months or less and those more than 12 months) and gender (males and females). To calculate the heterogeneity between studies, $t^{2}$ statistic and the $Q$ statistic were used. Publication bias was not estimated, because it is considered irrelevant for prevalence studies [26]. Moreover, due to different sensitivities and specificities of diagnostic methods, we assumed that our results would be "apparent" prevalence rates and did not represent true prevalence rates. Results are shown as forest plots of the pooled prevalence (with $95 \% \mathrm{CI}$ ) of microsporidia infection in dogs.

\section{Results}

\section{Study characteristics}

A flow diagram summarizing the study selection process is presented in Fig. 1. In brief, a total of 1431 records were found following the initial search of databases; after removing duplicates and/or non-eligible papers, 32 papers had eligibility to be included in this systematic review and meta-analysis [13, 23, 27-56]. Among these 32 studies, five studies had two datasets. In this regard, 32 studies containing 37 datasets were reviewed. The results of quality assessment according to JBI for eligible studies are mentioned in Tables 1, 3, and 4. The included articles in the present meta-analysis showed an acceptable quality. Out of the retrieved articles, 23, nine, and five datasets used molecular, serological, and microscopical methods, respectively. It should be noted that two studies used microscopic and coproantigen test for detection of microsporidia at the same time (the main characteristics of each study together with the types of implemented method are listed in Tables 1, 3 and 4, respectively). The results of the literature review and meta-analysis was separately considered for each of the three detection methods. Due to the low number of included studies in serological and microscopic methods, the subgroup analysis of continents and countries was performed only for molecular methods.

\section{The results based on molecular methods}

As shown in Table 1, a total of 23 datasets from 12 countries have examined the prevalence of microsporidia species in dogs using molecular methods including 11
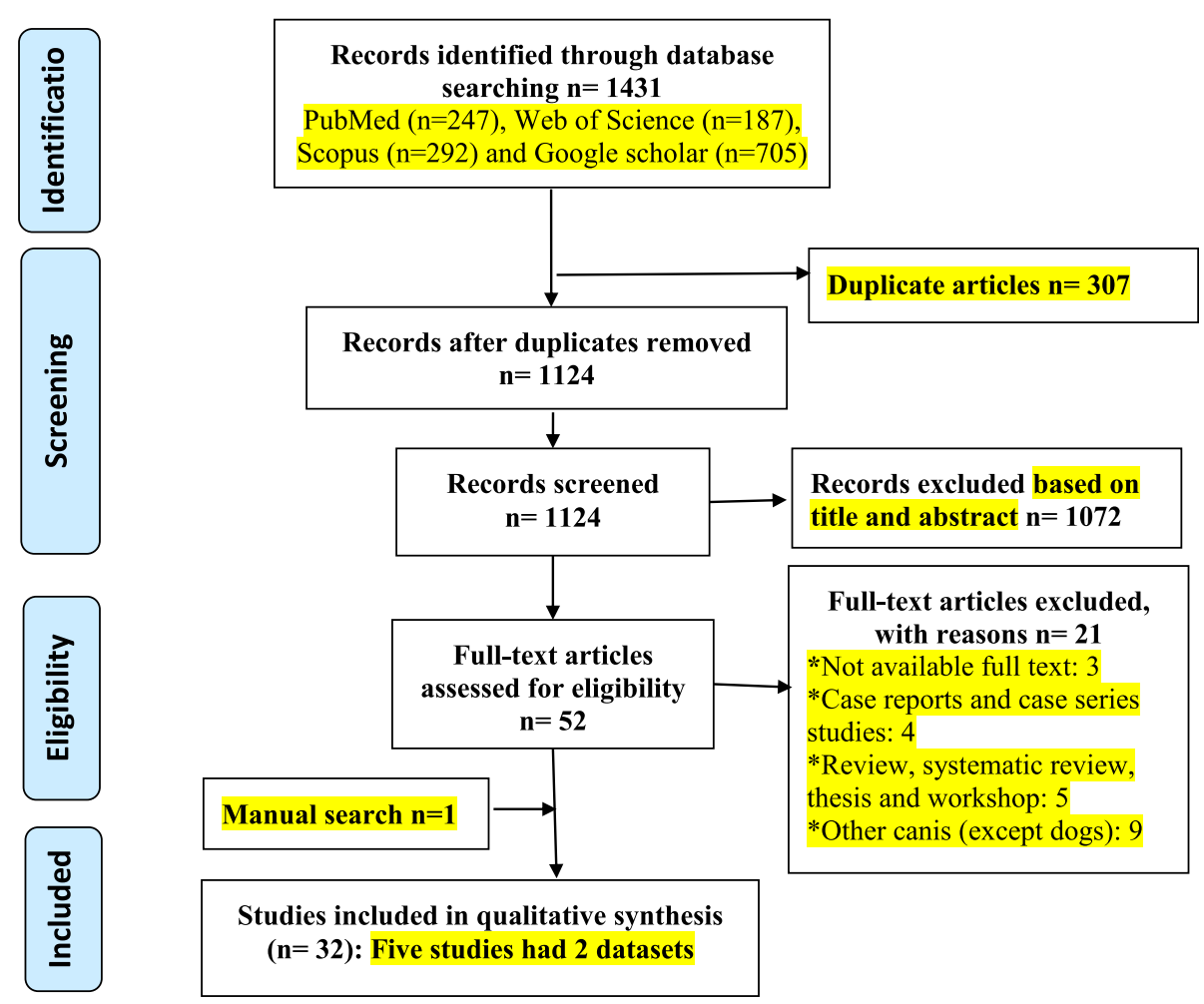

Fig. 1 PRISMA flow diagram describing included/excluded studies 


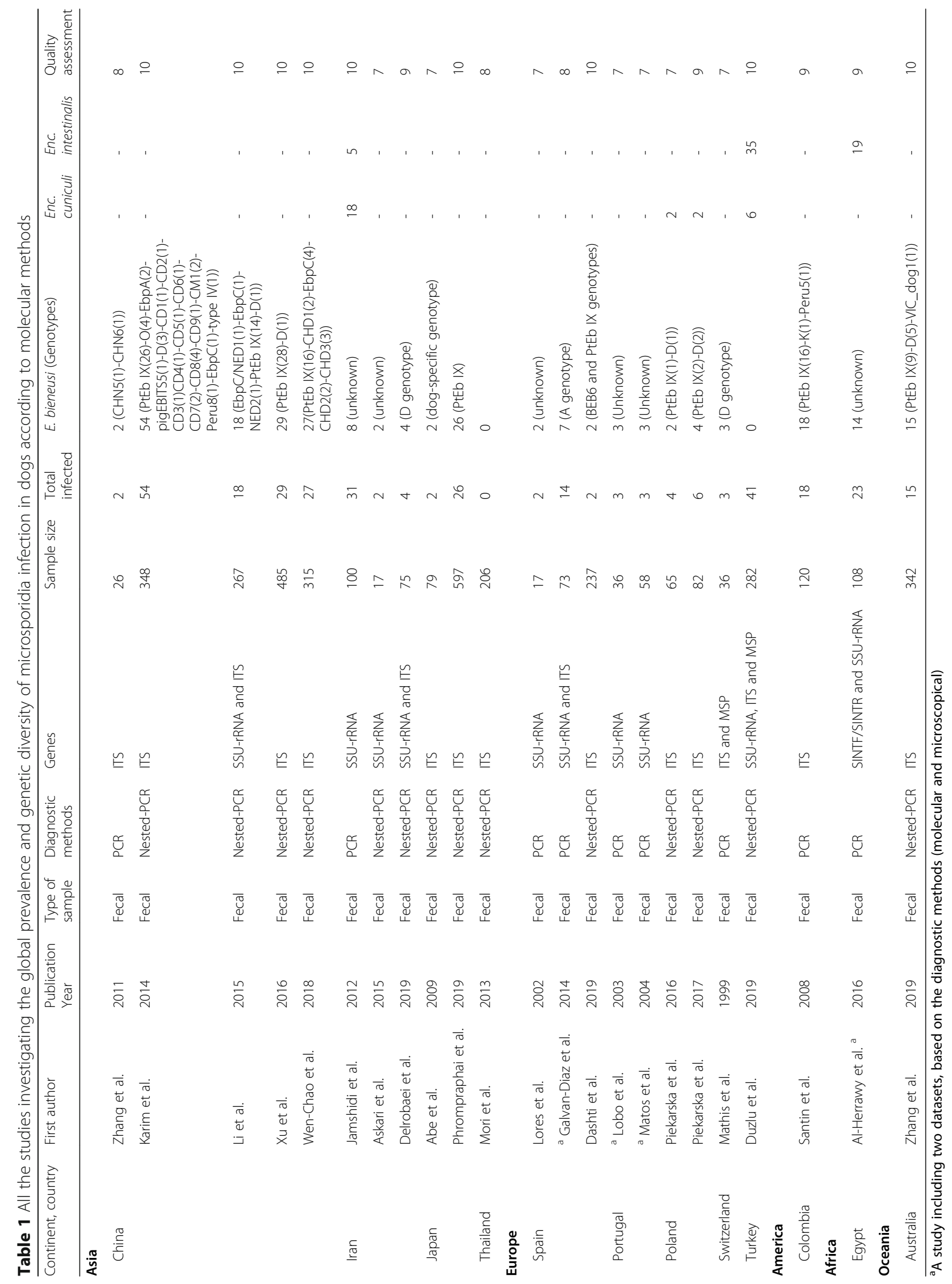


datasets (2515 animals) in Asia, nine datasets (886 animals) in Europe, one dataset (108 animals) in Africa, one dataset (120 animals) in America, and one dataset (342 animals) in Oceania. The countries with the highest number of published reports were China (five studies), Iran (three studies), and Spain (three studies). PCRbased methods were used in all molecular studies. Of the 23 included datasets, E. bieneusi $(21 / 23$; $91.30 \%$ studies), Enc. cuniculi (4/23; $17.39 \%$ studies), and Enc. intestinalis $(3 / 23 ; 13.04 \%$ studies) had the highest number of reports (Table 1). As shown in the Table 1, the internal transcribed spacer (ITS) gene was used to determine the genotypes of E. bieneusi (17/23 datasets; 73.91\%). In this regard, the PtEb IX (10 studies) and the D (eight studies) genotypes of $E$. bieneusi were the frequently reported genotypes (Table 1 ). The other reported E. bieneusi genotypes are shown in Table 1. Nevertheless, genotyping was not performed in six studies, because they used only the small subunit ribosomal RNA (SSU-rRNA) gene (Table 1 ). The pooled molecular prevalence of microsporidia infection in dogs thought to be $8.4 \%$ (95\% CI, 6.1-11.5\%; 329/3971) (Supplementary Figure 1). According to the identified species, the prevalences were 11.6\% (95\% CI: 6.7-19.2\%) for Enc. intestinalis (Supplementary Figure 2), 6.5\% (95\% CI: 4.9-8.7\%) for E. bieneusi (Fig. 2), and 4.5\% (95\% CI: 1.1-16.5\%) for Enc. cuniculi (Supplementary Figure 3). In the subgroup analysis regarding continents, the highest pooled prevalence was observed in Africa 21.3\%
(95\% CI, 14.6-30\%) followed by Europe 8.2\% (95\% CI, 513.2\%), Asia 7.7\% (95\% CI, 4.6-12.5\%), America 15\% (95\% CI, 9.7-22.6\%), and Oceania 4.4\% (95\% CI, 2.7-7.1\%) (Table 2). It should be noted that only one study has been conducted in America, Africa, and Oceania continents. In addition, the prevalence of microsporidia based on countries is categorized in Table 2.

\section{The results based on serological detection techniques}

A total of 1703 dogs from 9 datasets were evaluated for microsporidia sero-prevalence in dogs (Table 3). In this review, the ELISA (enzyme-linked immunosorbent assay) method was used in most of the datasets $(n=4)$, followed by IFA (immunofluorescence assay) method $(n=3)$, and DAT (direct agglutination test) method $(n=2)$. The included datasets represented seven different countries (i.e., three datasets from Slovakia, one dataset from Norway, one dataset from Colombia, one dataset from Brazil, one dataset from the USA, one dataset from Japan, and one dataset from Egypt). The pooled sero-prevalence of microsporidia in dogs was thought to be 20.9\% (95\% CI, 14.6-29\%) (Supplementary Figure 4).

\section{The results based on microscopic methods}

A total of five datasets from four different countries that used microscopic methods were included in the systematic review and meta-analysis; although in two studies,

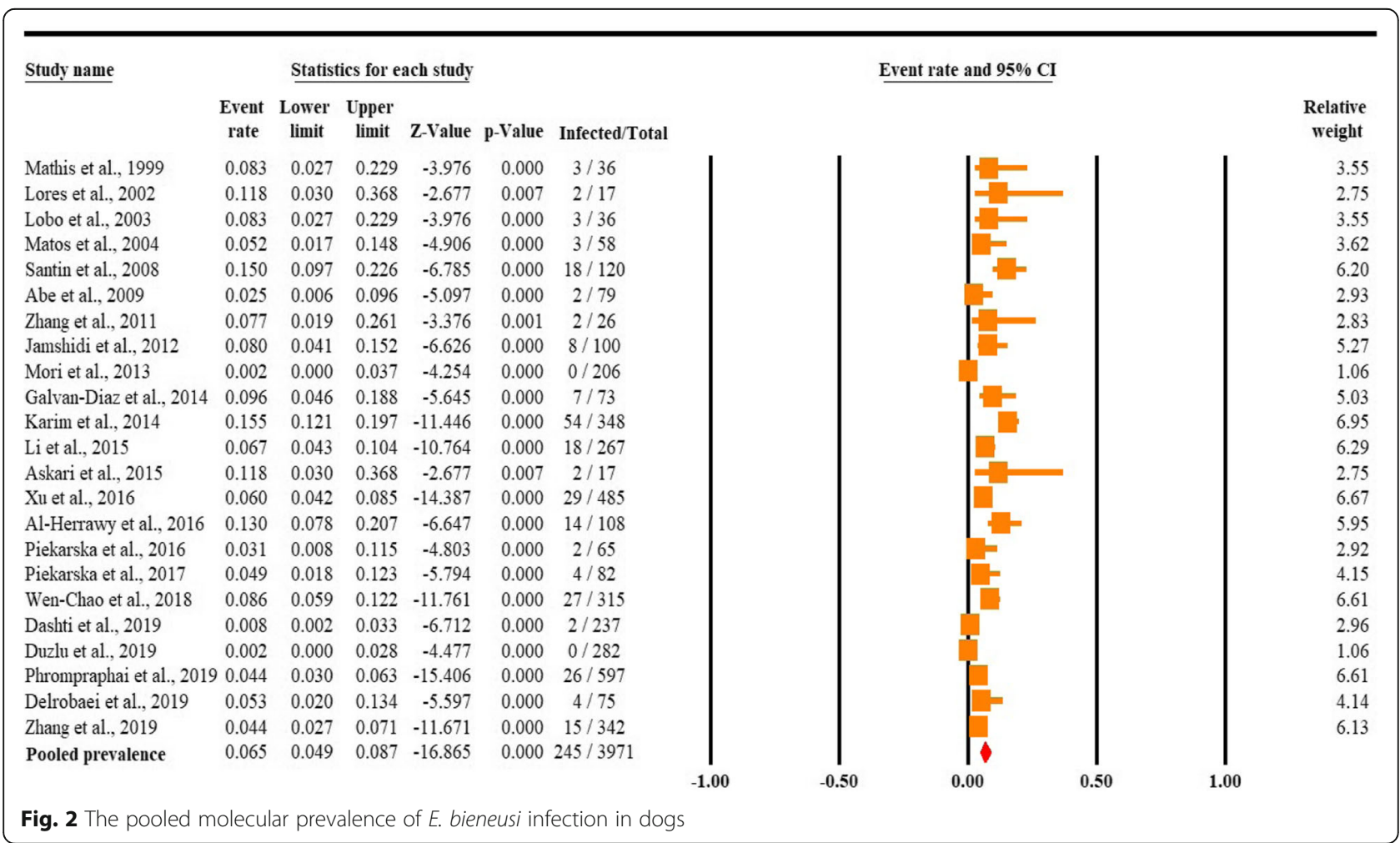


Table 2 Subgroup analysis of continents and countries based on molecular methods in dogs

\begin{tabular}{|c|c|c|c|c|}
\hline Continents/countries & Datasets $(n)$ & Sample size $(n)$ & Infected $(n)$ & Pooled prevalence \% (95\% Cl) \\
\hline Global & 23 & 3971 & 329 & $8.4(6.1-11.5)$ \\
\hline Asia & 11 & 2515 & 195 & $7.7(4.6-12.5)$ \\
\hline China & 5 & 1441 & 130 & $8.6(5.5-13.4)$ \\
\hline Iran & 3 & 192 & 37 & $13.7(3.5-40.9)$ \\
\hline Japan & 2 & 676 & 28 & $4.2(2.9-6)$ \\
\hline Thailand & 1 & 206 & 0 & 0 \\
\hline Europe & 9 & 886 & 78 & $8.2(5-13.2)$ \\
\hline Spain & 3 & 327 & 18 & $6.4(0.9-33.5)$ \\
\hline Portugal & 2 & 94 & 6 & $6.6(3-13.8)$ \\
\hline Poland & 2 & 147 & 10 & $6.8(3.7-12.2)$ \\
\hline Switzerland & 1 & 36 & 3 & $8.3(2.7-22.9)$ \\
\hline Turkey & 1 & 282 & 41 & $14.5(10.9-19.2)$ \\
\hline America & 1 & 120 & 18 & $15(9.7-22.6)$ \\
\hline Colombia & 1 & 120 & 18 & $15(9.7-22.6)$ \\
\hline Africa & 1 & 108 & 23 & $21.3(14.6-30)$ \\
\hline Egypt & 1 & 108 & 23 & $21.3(14.6-30)$ \\
\hline Oceania & 1 & 342 & 15 & $4.4(2.7-7.1)$ \\
\hline Australia & 1 & 342 & 15 & $4.4(2.7-7.1)$ \\
\hline
\end{tabular}

microscopy and coproantigen methods were used, the reported prevalence rates were same (Table 4). As shown in the Table 4, no countries were available for America and Oceania continents. The techniques used for this diagnostic method are listed in Table 4. The pooled prevalence of microsporidia in dogs (from five datasets including 355 dogs) was 23.1\% (95\% CI, 13.5$36.8 \%$ ) (Supplementary Figure 5).

\section{Confounding factors}

With regard to the type of dogs, infection rates were estimated to be $16 \%$ (95\% CI, 10.4-23.8\%) in unknown dogs, $12.8 \%$ (95\% CI, 8.2-19.4\%) in stray dogs, and $11.2 \%$ (95\% CI, 7.9-15.8\%) in pet (domesticated) dogs (Table 5 and Supplementary Table 1). Male dogs and age > 12 months had a higher infection rate; however, these were not statistically significant (Table 5 and Supplementary Table 2).

\section{Discussion}

Over the years, zoonotic transmission of various species of microsporidia and the role of animals as reservoir for human infection are important issues in medical and veterinary practices $[57,58]$. Dogs are considered one of the potential reservoirs for human-infecting microsporidia. According to our results, the prevalence of infection in the microscopic, serological, and molecular methods was estimated at $23.1 \%, 20.9 \%$, and $8.4 \%$, respectively. One of the most important reasons for the difference in prevalence is that each of the studied methods has its

Table 3 All the studies investigating the global prevalence of microsporidia in dogs according to serological methods

\begin{tabular}{|c|c|c|c|c|c|c|c|}
\hline First author & Publication year & Country & Type of sample & Diagnostic methods & Sample size & Infected & Quality assessment \\
\hline Stefkovic & 2001 & Slovakia & Serum & IFA & 178 & 53 & 7 \\
\hline Akerstedt & 2003 & Norway & Serum & IFA & 237 & 0 & 10 \\
\hline Halanova et al. & 2003 & Slovakia & Serum & IFA & 193 & 73 & 10 \\
\hline Lindsay et al. ${ }^{a}$ & 2009 & Brazil & Serum & DAT & 113 & 27 & 10 \\
\hline Lindsay et al. ${ }^{a}$ & 2009 & Colombia & Serum & DAT & 254 & 19 & 10 \\
\hline Malcekova et al. & 2010 & Slovakia & Serum & ELISA & 111 & 17 & 10 \\
\hline Sasaki et al. & 2011 & Japan & Serum & ELISA and WB & 472 & 103 & 10 \\
\hline Cray and Rivas & 2013 & USA & Serum & ELISA & 125 & 27 & 9 \\
\hline Abu-Akkada et al. & 2015 & Egypt & Serum & ELISA & 20 & 8 & 8 \\
\hline
\end{tabular}

IFA immunofluorescence assay, DAT direct agglutination test, ELISA Enzyme-linked immunosorbent assay, WB western blot

${ }^{a} \mathrm{~A}$ study including two datasets, based on the countries 
Table 4 All the studies investigating the global prevalence of microsporidia in dogs according to microscopy or coproantigens detection methods

\begin{tabular}{llllllll}
\hline First author & Publication year & Country & Type of sample & Diagnostic methods & Sample size & Infected & Quality assessment \\
\hline Lobo et al. $^{\text {a }}$ & 2003 & Portugal & Fecal & MTS and IFA & 36 & 5 & 7 \\
Matos et al. $^{\text {a }}$ & 2004 & Portugal & Fecal & MTS and IFA ${ }^{\text {b }}$ & 58 & 9 & 7 \\
Al-Sadi et al. & 2013 & Iraq & Fecal & Multiple staining ${ }^{\text {c }}$ & 80 & 11 & 8 \\
Galvan-Diaz et al. $^{\text {a }}$ & 2014 & Spain & Fecal & WS & 73 & 32 & 8 \\
Al-Herrawy et al. $^{\text {a }}$ & 2016 & Egypt & Fecal & MTS & 108 & 36 & 9 \\
\hline
\end{tabular}

MTS modified trichrome staining, IFA immunofluorescence assay for coproantigens survey, WS Weber's staining

${ }^{a} A$ study including two datasets, based on the diagnostic methods (molecular and microscopical)

${ }^{\mathrm{b}} \mathrm{MTS}$ and IFA: both methods reported the same prevalence

${ }^{c}$ Multiple staining: Giemsa, quick-hot Gram-chromotrope, Weber-green modified trichrome, Ryan-blue modified trichrome, and Calcofluor white stains

own sensitivity and specificity [59, 60]. Traditionally, light microscopy using specific staining techniques is one of the ways to diagnose microsporidia infection [61]. However, this method is difficult and needs a wellexperienced person due to its similarity to bacteria or yeasts in stool samples [31, 62]. In the current study, the results of the microscopic prevalence are higher than the other two methods, which may be due to misdiagnosis with other elements and microorganisms. Nevertheless, nowadays molecular techniques are considered the gold standard for detection and differentiation of microsporidia at the species/genotype levels $[63,64]$. The main advantage of molecular methods is higher sensitivity and specificity, along with easier interpretation $[65,66]$. In this regard, the pooled prevalence obtained by molecular methods (8.4\%) can be considered as "true" prevalence. On the other hand, although serological techniques (i.e., DAT, IFA, and ELISA) are more sensitive than the microscopic methods, high costs and false-positive reactions are considered disadvantages. In addition, there is no reliable serological method for detection of E. bieneusi [36, 67].

According to the molecular analysis, E. bieneusi is a complex species with numerous genotypes, diverse host spectrum and pathogenicity, and wide distribution [6870]. The genetic data on $E$. bieneusi diversity rely almost exclusively on the analysis of the ITS region, which provides valuable information about the transmission and pathogenic potential of this parasite [70]. As the molecular results of this review showed, most of the reports were related to E. bieneusi the genotypes PtEb IX and $\mathrm{D}$. In this regard, the genotype PtEb IX, a dogspecific genotype, could have an exclusive dog-to-dog transmission that may cause some gastrointestinal disorders in dogs $[12,71]$. As shown in Table 1, zoonotic transmission between dogs and humans is possible for the genotypes D, A, Peru 5, Peru 8, and type IV that most of these genotypes have been reported in immunocompromised individuals [5, 70, 72-75]. This finding proposed that dogs and humans can either infect each other or be infected from the same environmental sources (i.e., water sources and vegetables). However, more samples from these animals and humans should be genotyped to confirm the zoonotic transmission of these genotypes.

According to formal reports, the approximate population of pet and stray dogs has been estimated at 471 million and 200 million worldwide, respectively [76] (https://www.statista.com/statistics/1044386/dog-andcat-pet-population-worldwide/) (https://www.npr.org/2 017/12/29/574598877/no-easy-answer-to-growing-number-of-stray-dogs-in-the-u-s-advocate-says). In this regard, the top 10 countries with the largest population of dogs include the USA, Brazil, China, Russia, Japan, Philippines, India, Argentina, UK, and France (https:// www.mapsofworld.com/world-top-ten/countries-withmost-pet-dog-population.html). In this review, most studies have been conducted in developed countries,

Table 5 Subgroup analysis of animal type, age and gender in dogs

\begin{tabular}{|c|c|c|c|c|c|}
\hline Variables & & Sample size $(n)$ & Infected $(n)$ & Pooled prevalence \% (95\% Cl) & Odds ratio $(\mathrm{OR})(95 \% \mathrm{Cl})$ \\
\hline \multirow[t]{3}{*}{ Type of dogs } & Pet (domestic) dogs & 2847 & 287 & $11.2(7.9-15.8)$ & - \\
\hline & Stray dogs & 917 & 126 & $12.8(8.2-19.4)$ & \\
\hline & Unknown & 2083 & 336 & $16(10.4-23.8)$ & \\
\hline \multirow[t]{2}{*}{ Age } & $\leq 12$ months & 339 & 22 & $8.3(2.8-21.9)$ & $0.76(0.23-2.48)$ \\
\hline & $>12$ months & 1177 & 98 & $10.5(6.2-17)$ & \\
\hline \multirow[t]{2}{*}{ Gender } & Male & 783 & 104 & $13.8(9.2-20.1)$ & $1.08(0.74-1.59)$ \\
\hline & Female & 569 & 62 & 11.9 (7.9-17.5) & \\
\hline
\end{tabular}


especially European countries, where laboratory tools are easily accessible compared to developing countries. Therefore, some key countries (countries with the highest number of dogs) have few or no studies, and the need for further studies and more attention to microsporidia infection in dogs in these countries is emphasized.

The results of this meta-analysis showed that the prevalence of microsporidia infection in stray dogs were higher than that in pet (domesticated) dogs. Since stray dogs are freely scattered in the environment and easily access various environmental sources, they can be considered an important reservoir of microsporidia. As a result, it is recommended that health officials and health policymakers provide health strategies to prevent stray dogs from entering urban and rural areas in order to control infections.

In the current meta-analyses, we observed a higher microsporidia prevalence in dogs aged $>12$ months and male dogs, but these were not statistically significant. One of the reasons for the higher prevalence of the infection in older age can be related to the higher risk of exposure to microsporidia.

As in our previous meta-analysis studies [19, 77, 78], the strengths of this study include the rigorous methodology, the comprehensive literature search in four international databases, the large total sample size, and analysis of several subgroups. Moreover, this study has some limitations and the results presented here should be interpreted with regard to these limitations. Limitations compose inclusion of reports with a lack of information on age, gender, symptoms, and risk factors; failed online registration (PROSPERO) due to the data were already extracted, some reports with low sample size, variations in the sensitivity and specificity of diagnostic methods, and the possibility of missing some studies during search strategy. Because of these limitations, it should be noted that our results may not reflect the true prevalence, and the reported numbers are apparent prevalence. Nevertheless, we believe what we report here is very close to true microsporidia prevalence in dogs from a global perspective.

\section{Conclusion}

We have found that there is a high burden of microsporidia infection in dogs. Moreover, our results have shown that E. bieneusi the genotypes PtEb IX and D are the common species/genotypes in dogs. These data should be taken into consideration by the health authorities, physicians, veterinarians, and dog's owners. Furthermore, this study signifies the importance of periodical monitoring of dogs for microsporidia. We suggest additional investigations to further clarify the prevalence and genetic diversity of microsporidiosis in the world to guide the development of appropriate public health interventions.

\section{Supplementary information}

Supplementary information accompanies this paper at https://doi.org/10. 1186/s41182-020-00265-0.

Additional file 1: Supplementary Figure 1. The pooled molecular prevalence of microsporidia infection in dogs. Supplementary Figure 2. The pooled molecular prevalence of Enc. intestinalis infection in dogs. Supplementary Figure 3. The pooled molecular prevalence of Enc. cuniculi infection in dogs. Supplementary Figure 4. The pooled seroprevalence of microsporidia infection in dogs. Supplementary Figure 5. The pooled prevalence of microsporidia infection in dogs.

Additional file 2: Supplementary Table 1. Excel spreadsheet of extracted data from included studies for type of dogs.

Additional file 3: Supplementary Table 2. Excel spreadsheet of extracted data from included studies for age and gender of dogs.

\section{Acknowledgements}

The authors are very grateful to Dr. Hamed Mirjalali (Shahid Beheshti University of Medical Sciences, Tehran, Iran) for her kind assistance during the preparation of this manuscript.

\section{Authors' contributions}

All authors contributed to study design. AT contributed to all parts of the study. AT, SB, and SK contributed to study implementation. AT collaborated in the analysis and interpretation of data. AT collaborated in the manuscript writing and revision. All the authors commented on the drafts of the manuscript and approved the final version of the article.

\section{Funding}

This research did not receive any specific grant from funding agencies in public, commercial, or not-for-profit sectors.

Availability of data and materials

All data generated or analyzed during study are included in this systematic review and meta-analysis

Ethics approval and consent to participate Not applicable

\section{Consent for publication}

Not applicable

\section{Competing interests}

None declared.

Received: 10 June 2020 Accepted: 24 August 2020

Published online: 04 September 2020

References

1. Han B, Weiss LM. Microsporidia: Obligate intracellular pathogens within the fungal kingdom. Microbiol Spectr. 2017;5(2):97-113.

2. Stentiford G, Becnel J, Weiss LM, Keeling P, Didier E, Bjornson S, Freeman M, Brown M, Roesel K, Sokolova Y. Microsporidia-emergent pathogens in the global food chain. Trend Parasitol. 2016;32(4):336-48.

3. Brown MJ. Microsporidia: an emerging threat to bumblebees? Trend Parasitol. 2017;33(10):754-62.

4. Henriques-Gil N, Haro M, Izquierdo F, Fenoy S, del Águila C. Phylogenetic approach to the variability of the microsporidian Enterocytozoon bieneusi and its implications for inter-and intrahost transmission. Appl Environ Microbiol. 2010;76(10):3333-42.

5. Karimi K, Mirjalali H, Niyyati M, Haghighi A, Pourhoseingholi MA, Sharifdini M, Naderi N, Zali MR. Molecular epidemiology of Enterocytozoon bieneusi and Encephalitozoon sp., among immunocompromised and immunocompetent subjects in Iran. Microb Pathog. 2020;141:103988.

6. Sak B, Kváč M, Kučerová Z, Květoňová D, Saková K. Latent microsporidial infection in immunocompetent individuals - a longitudinal study. PLoS Negl Trop Dis. 2011;5(5):e1162. https://doi.org/10.1371/journal.pntd.0001162.

7. Weber R, Kuster H, Visvesvara GS, Bryan RT, Schwartz DA, Lüthy R. Disseminated microsporidiosis due to Encephalitozoon hellem: pulmonary 
colonization, microhematuria, and mild conjunctivitis in a patient with AIDS. Clin Infect Dis. 1993;17(3):415-9.

8. Nooshadokht M, Sharifi I, Mohammadi MA, Pirestani M, Afgar A, Mahootchi A, Salari S. Intestinal microsporidiosis in Iran: infection in immunecompromised and immunocompetent patients. Curr med mycol. 2017;3(1): 30.

9. Taghipour A, Tabarsi P, Sohrabi MR, Riahi SM, Rostami A, Mirjalali H, Malih N, Haghighi A. Frequency, associated factors and clinical symptoms of intestinal parasites among tuberculosis and non-tuberculosis groups in Iran: a comparative cross-sectional study. Trans R Soc Trop Med Hyg. 2019;113(5): 234-41.

10. Didier E, Stovall M, Green L, Brindley P, Sestak K, Didier P. Epidemiology of microsporidiosis: sources and modes of transmission. Vet Parasitol. 2004; 126(1-2):145-66.

11. Javanmard E, Mirjalali H, Niyyati M, Jalilzadeh E, Seyed Tabaei SJ, Asadzadeh Aghdaei H, Nazemalhosseini-Mojarad E, Zali MR. Molecular and phylogenetic evidences of dispersion of human-infecting microsporidia to vegetable farms via irrigation with treated wastewater: One-year follow up. Int J Hyg Environ Health. 2018;221(4):642-51.

12. Santín M, Fayer R. Microsporidiosis: Enterocytozoon bieneusi in domesticated and wild animals. Res vet sci. 2011;90(3):363-71.

13. Askari Z, Mirjalali H, Mohebali M, Zarei Z, Shojaei S, Rezaeian T, Rezaeian M Molecular detection and identification of zoonotic microsporidia spore in fecal samples of some animals with close-contact to human. Iran J Parasitol. 2015:10(3):381.

14. Coppinger R, Schneider R. The evolution of working dogs. In: Serpell J, editor. The domestic dog: its evolution, behaviour and interactions with people. New York: Cambridge University Press; 1995. p. 21-47.

15. Beck AM, Meyers NM. Health enhancement and companion animal ownership. Annu Rev public health. 1996;17(1):247-57.

16. Macpherson CN. Dogs, zoonoses and public health. Wallingford: CABI Publishing; 2012

17. Rostami A, Riahi SM, Hofmann A, Ma G, Wang T, Behniafar H, Taghipour A, Fakhri Y, Spotin A, Chang BC. Global prevalence of Toxocara infection in dogs. Adv Parasitol. 2020;109:561.

18. Paul M, King L, Carlin EP. Zoonoses of people and their pets: a US perspective on significant pet-associated parasitic diseases. Trend Parasitol. 2010;26(4):153-4.

19. Taghipour A, Olfatifar M, Bahadory S, Godfrey SS, Abdoli A, Khatami A, Javanmard E, Shahrivar F. The global prevalence of Cryptosporidium infection in dogs: a systematic review and meta-analysis. Vet Parasitol. 2020;281:109093.

20. Kelly TR, Machalaba C, Karesh WB, Crook PZ, Gilardi K, Nziza J, Uhart MM Robles EA, Saylors K, Joly DO. Implementing One Health approaches to confront emerging and re-emerging zoonotic disease threats: lessons from PREDICT. One Health Outlook. 2020;2(1):1-7.

21. Atlas R, Rubin C, Maloy S, Daszak P, Colwell R, Hyde B. One health-attaining optimal health for people, animals, and the environment. Microbe. 2010;5(9):383-9.

22. Tosoni A, Nebuloni M, Ferri A, Bonetto S, Antinori S, Scaglia M, Xiao L, Moura H, Visvesvara GS, Vago L. Disseminated microsporidiosis caused by Encephalitozoon cuniculi III (dog type) in an Italian AIDS patient: a retrospective study. Mod pathol. 2002;15(5):577-83.

23. Delrobaei M, Jamshidi S, Shayan P, Ebrahimzade E, Tamai IA, Rezaeian M, Mirjalali $\mathrm{H}$. Molecular detection and genotyping of intestinal microsporidia from stray dogs in Iran. Iran J Parasitol. 2019;14(1):159.

24. Moher D, Shamseer L, Clarke M, Ghersi D, Liberati A, Petticrew M, Shekelle P, Stewart LA. Preferred reporting items for systematic review and metaanalysis protocols (PRISMA-P) 2015 statement. Syst Rev. 2015;4(1):1.

25. Institute JB. Joanna Briggs Institute reviewers' manual: 2014 edition. Australia: The Joanna Briggs Institute; 2014.

26. Hunter JP, Saratzis A, Sutton AJ, Boucher RH, Sayers RD, Bown MJ. In metaanalyses of proportion studies, funnel plots were found to be an inaccurate method of assessing publication bias. J Clin Epidemiol. 2014;67(8):897-903.

27. Abu-Akkada SS, Ashmawy KI, Dweir AW. First detection of an ignored parasite, Encephalitozoon cuniculi, in different animal hosts in Egypt. Parasitol Res. 2015;114(3):843-50.

28. Al-Herrawy AZ. Microsporidial spores in fecal samples of some domesticated animals living in Giza. Egypt. Iran J Parasitol. 2016;11(2):195.

29. Mathis A, Breitenmoser A, Deplazes P. Detection of new Enterocytozoon genotypes in faecal samples of farm dogs and a cat. Parasite. 1999;6(2): 189-93.
30. Stefkovic M, Valencakova A, Balent P, Maslej P, Halanova M. A study on antibody prevalence due to microsporidian Encephalitozoon cuniculi in dogs (Canis familiaris) using indirect immunofluorescence. Folia Vete (Slovak Republic). 2001:343-50.

31. Lores B, Del Aguila C, Arias C. Enterocytozoon bieneusi (microsporidia) in faecal samples from domestic animals from Galicia. Spain. Mem Inst Oswaldo Cruz. 2002;97(7):941-5.

32. Åkerstedt J. Serological investigation of canine encephalitozoonosis in Norway. Parasitol Res. 2002;89(1):49-52.

33. Halanova M, Cislakova L, Valencakova A, Balent P, Adam J, Travnicek M. Serological screening of occurrence of antibodies to Encephalitozoon cuniculi in humans and animals in eastern Slovakia. Ann Agric Environ Med. 2003;10(1):117-20.

34. Lobo ML, Teles A, Da Cunha MB, Henriques J, Lourenço AM, Antunes F, Matos O. Microsporidia detection in stools from pets and animals from the zoo in Portugal: a preliminary study. J Eukaryot Microbiol. 2003;50(6):581-2.

35. Matos $\mathrm{O}$, Lobo ML, Teles $\mathrm{A}$, Antunes F. Is microsporidial infection in animals a potential source for human microsporidiosis. Southeast Asian J Trop Med Public Health. 2004;35:48-53.

36. Malčeková B, Halánová M, Sulínová Z, Molnár L, Ravaszová P, Adam J, Halán M, Valocký I, Baranovič M. Seroprevalence of antibodies to Encephalitozoon cuniculi and Encephalitozoon intestinalis in humans and animals. Res Vet Sci. 2010;89(3):358-61.

37. Galván-Díaz AL, Magnet A, Fenoy S, Henriques-Gil N, Haro M, Gordo FP, Miro G, del Aguila C, Izquierdo F. Microsporidia detection and genotyping study of human pathogenic E. bieneusi in animals from Spain. PLoS One. 2014;9(3):e106017. https://doi.org/10.1371/journal.pone.0106017.

38. Piekarska J, Kicia M, Wesołowska M, Kopacz Ż, Gorczykowski M, Szczepankiewicz B, Kváč M, Sak B. Zoonotic microsporidia in dogs and cats in Poland. Vet Parasitol. 2017;246:108-11.

39. Pekmezci D, Pekmezci GZ, Yildirim A, Duzlu O, Inci A. Molecular detection of zoonotic microsporidia in domestic cats in Turkey: a preliminary study. Acta Parasitol. 2019;64(1):13-8.

40. Dashti A, Santín M, Cano L, de Lucio A, Bailo B, de Mingo MH, Köster PC, Fernández-Basterra JA, Aramburu-Aguirre J, López-Molina N. Occurrence and genetic diversity of Enterocytozoon bieneusi (Microsporidia) in owned and sheltered dogs and cats in Northern Spain. Parasitol Res. 2019;118(10): 2979-87.

41. Abe N, Kimata I, Iseki M. Molecular evidence of Enterocytozoon bieneusi in Japan. J Vet Med Sci. 2009;71(2):217-9.

42. Zhang $X$, Wang Z, Su Y, Liang X, Sun X, Peng S, Lu H, Jiang N, Yin J, Xiang M. Identification and genotyping of Enterocytozoon bieneusi in China. J Clin Microbiol. 2011;49(5):2006-8.

43. Jamshidi S, Tabrizi AS, Bahrami M, Momtaz H. Microsporidia in household dogs and cats in Iran; a zoonotic concern. Vet Parasitol. 2012;185(2-4):121-3.

44. Karim MR, Dong H, Yu F, Jian F, Zhang L, Wang R, Zhang S, Rume FI, Ning C, Xiao L. Genetic diversity in Enterocytozoon bieneusi isolates from dogs and cats in China: host specificity and public health implications. J Clin Microbiol. 2014;52(9):3297-302.

45. Li W, Li Y, Song M, Lu Y, Yang J, Tao W, Jiang Y, Wan Q, Zhang S, Xiao L. Prevalence and genetic characteristics of Cryptosporidium, Enterocytozoon bieneusi and Giardia duodenalis in cats and dogs in Heilongjiang province. China. Vet Parasitol. 2015;208(3-4):125-34.

46. Xu H, Jin Y, Wu W, Li P, Wang L, Li N, Feng Y, Xiao L. Genotypes of Cryptosporidium spp., Enterocytozoon bieneusi and Giardia duodenalis in dogs and cats in Shanghai, China. Parasites Vectors. 2016;9(1):121.

47. Wen-Chao L, Jie Q, Kai W, You-Fang G. Genotypes of Enterocytozoon bieneusi in dogs and cats in eastern China. Iran J Parasitol. 2018;13(3):457.

48. Phrompraphai T, Itoh N, lijima Y, Ito Y, Kimura Y. Molecular detection and genotyping of Enterocytozoon bieneusi in family pet dogs obtained from different routes in Japan. Parasitol Int. 2019;70:86-8.

49. Lindsay DS, Goodwin DG, Zajac AM, Cortés-Vecino J, Gennar S, Rosypal AC, Dubey J. Serological survey for antibodies to Encephalitozoon cuniculi in ownerless dogs from urban areas of Brazil and Colombia. J Parasitol. 2009; 95(3):760-3.

50. Cray C, Rivas Y. Seroprevalence of Encephalitozoon cuniculi in dogs in the United States. J Parasitol. 2013;99(1):153-4.

51. Zhang Y, Koehler AV, Wang T, Cunliffe D, Gasser RB. Enterocytozoon bieneusi genotypes in cats and dogs in Victoria. Australia. BMC Microbiol. 2019;19(1):183.

52. Santín M, Vecino JAC, Fayer R. Enterocytozoon bieneusi genotypes in dogs in Bogota. Colombia. Am J Trop Med Hyg. 2008;79(2):215-7. 
53. Piekarska J, Kicia M, Wesolowska M, Kopacz Z, Gorczykowski M, Sobieraj B, Kvac M, Sak B. Human-pathogenic microsporidia in household dogs and cats in Wroclaw (Poland). Ann Parasitol. 2016;62:124.

54. Mori H, Mahittikorn A, Thammasonthijarern N, Chaisiri K, Rojekittikhun W, Sukthana Y. Presence of zoonotic Enterocytozoon bieneusi in cats in a temple in central Thailand. Vet Parasitol. 2013;197(3-4):696-701.

55. Al-Sadi H, Al-Mahmood S. Microsporidial infection in some domestic and laboratory animals in Iraq. Int J Basic Appl Med Sci. 2013;3(3):78-91.

56. Sasaki M, Yamazaki A, Haraguchi A, Tatsumi M, Ishida K, Ikadai H. Serological survey of Encephalitozoon cuniculi infection in Japanese dogs. J Parasitol. 2011;97(1):167-9.

57. Mathis A, Weber R, Deplazes P. Zoonotic potential of the microsporidia. Clin Microbiol Rev. 2005;18(3):423-45.

58. Javanmard E, Nemati S, Sharifdini M, Rostami A, Mirjalali H, Zali MR. The first report and molecular analysis of Enterocytozoon bieneusi from raccoon (Procyon lotor) in North of Iran. J Eukaryot Microbiol. 2020;67(3):359-68.

59. Garcia LS. Laboratory identification of the microsporidia. J Clin Microbiol 2002;40(6):1892-901

60. Franzen C, Müller A. Microsporidiosis: human diseases and diagnosis. Microb Infect. 2001;3(5):389-400.

61. Didier ES, Orenstein JM, Aldras A, Bertucci D, Rogers LB, Janney FA. Comparison of three staining methods for detecting microsporidia in fluids. J Clin Microbiol. 1995;33(12):3138-45.

62. Field A, Marriott D, Hing M. The Warthin-Starry stain in the diagnosis of small intestinal microsporidiosis in HIV-infected patients. Folia Parasito (Praha). 1993;40(4):261-6

63. Wang S-N, Sun Y, Zhou H-H, Lu G, Qi M, Liu W-S, Zhao W. Prevalence and genotypic identification of Cryptosporidium spp. and Enterocytozoon bieneusi in captive Asiatic black bears (Ursus thibetanus) in Heilongjiang and Fujian provinces of China. BMC Vet Res. 2020;16(1):1-7.

64. Sulaiman IM, Fayer R, Yang C, Santin M, Matos O, Xiao L. Molecular characterization of Enterocytozoon bieneusi in cattle indicates that only some isolates have zoonotic potential. Parasitol Res. 2004;92(4):328-34.

65. Morio F, Poirier P, Le Govic Y, Laude A, Valot S, Desoubeaux G, Argy N, Nourrisson C, Pomares C, Machouart M. Assessment of the first commercial multiplex PCR kit (ParaGENIE Crypto-Micro Real-Time PCR) for the detection of Cryptosporidium spp., Enterocytozoon bieneusi, and Encephalitozoon intestinalis from fecal samples. Diag Microbiol Infect Dis. 2019;95(1):34-7.

66. Ghoyounchi R, Mahami-Oskouei M, Rezamand A, Spotin A, Aminisani N, Nami S, Pirestani M, Berahmat R, Madadi S. Molecular phylodiagnosis of Enterocytozoon bieneusi and Encephalitozoon intestinalis in children with cancer: microsporidia in malignancies as an emerging opportunistic infection. Acta Parasitol. 2019;64(1):103-11.

67. Ashmawy K, Abuakkada S, Awad A. Seroprevalence of antibodies to Encephalitozoon cuniculi and Toxoplasma gondii in farmed domestic rabbits in Egypt. Zoonoses Public Health. 2011;58(5):357-64.

68. Didier ES. Microsporidiosis: an emerging and opportunistic infection in humans and animals. Acta Trop. 2005;94(1):61-76.

69. Matos O, Lobo ML, Xiao L. Epidemiology of Enterocytozoon bieneusi infection in humans. J Parasitol Res. 2012;2012:1-19.

70. Thellier M, Breton J. Enterocytozoon bieneusi in human and animals, focus on laboratory identification and molecular epidemiology. EDP Sciences. 2008. p. 349-58.

71. Santin M, Fayer R. Enterocytozoon bieneusi genotype nomenclature based on the internal transcribed spacer sequence: a consensus. J Eukaryot Microbiol. 2009;56(1):34-8.

72. Sulaiman IM, Bern C, Gilman R, Cama V, Kawai V, Vargas D, Ticona E, Vivar A, Lxiao L. A molecular biologic study of Enterocytozoon bieneusi in HIVinfected patients in Lima, Peru. J Eukaryot Microbiol. 2003;50:591-6.

73. Liguory O, Sarfati C, Derouin F, Molina J-M. Evidence of different Enterocytozoon bieneusi genotypes in patients with and without human immunodeficiency virus infection. J Clin Microbiol. 2001;39(7):2672-4.

74. Wang L, Zhang H, Zhao X, Zhang L, Zhang G, Guo M, Liu L, Feng Y, Xiao L. Zoonotic Cryptosporidium species and Enterocytozoon bieneusi genotypes in HIV-positive patients on antiretroviral therapy. J Clin Microbiol. 2013;51(2): 557-63.

75. Mirjalali H, Mirhendi H, Meamar AR, Mohebali M, Askari Z, Mirsamadi ES, Rezaeian M. Genotyping and molecular analysis of Enterocytozoon bieneusi isolated from immunocompromised patients in Iran. Infect Genet Evol. 2015;36:244-9.
76. Lyu P. Proposal on solutions to stray dog problem in American cities. J Pol Sci Public Aff. 2015;3:175.

77. Taghipour A, Rostami A, Sepidarkish M, Ghaffarifar F. Is Ascaris lumbricoides a risk factor for development of asthma? A systematic review and metaanalysis. Microb Pathog. 2020;142:104099.

78. Taghipour A, Mosadegh M, Kheirollahzadeh F, Olfatifar M, Safari H, Nasiri MJ, Fathi A, Badri M, Dogaheh HP, Azimi T. Are intestinal helminthsplaying a positive role in tuberculosis risk? A systematic review and meta-analysis. PloS one. 2019;14(10):e0223722. https://doi.org/10.1371/journal.pone. 0223722 .

\section{Publisher's Note}

Springer Nature remains neutral with regard to jurisdictional claims in published maps and institutional affiliations.
Ready to submit your research? Choose BMC and benefit from:

- fast, convenient online submission

- thorough peer review by experienced researchers in your field

- rapid publication on acceptance

- support for research data, including large and complex data types

- gold Open Access which fosters wider collaboration and increased citations

- maximum visibility for your research: over $100 \mathrm{M}$ website views per year

At BMC, research is always in progress.

Learn more biomedcentral.com/submissions 